\title{
PSICOLOGÍA COMUNITARIA, PROGRAMAS SOCIALES Y NEOLIBERALISMO: LA EXPERIENCIA CHILENA
}

\author{
Héctor Berroeta ${ }^{1}$ \\ Universidad de Valparaíso, Chile
}

María Isabel Reyes

Pontificia Universidad Católica de Valparaíso, Chile

Bárbara Olivares

Universidad Diego Portales, Chile

\section{María Inés Winkler}

Universidad de Santiago de Chile, Chile

\section{Isaac Prilleltensky}

University of Miami, USA

\section{RESUMEN}

El antagonismo valórico entre la Psicología Comunitaria (PC) y el Neoliberalismo condiciona el quehacer profesional de la PC. Con el objetivo de conocer el modo en que se despliega la tensión entre políticas neoliberales y psicología comunitaria, se describe y analiza las formas en que se integra la perspectiva comunitaria en un conjunto de programas gubernamentales en Chile, que se declaran "comunitarios". Los resultados muestran que los programas de políticas públicas revisados, no cumplen con las posibilidades de la PC para fortalecer las comunidades. Se evidencia la urgente necesidad de recuperar y fortalecer la consideración de la dimensión ética- política en la formación profesional y la participación de la $\mathrm{PC}$ en políticas públicas.

\section{Palabras Claves}

programas sociales, neoliberalismo, psicología comunitaria

\begin{abstract}
The value antagonism between Community Psychology (PC) and Neoliberalism conditions professional work. In order to know the way in which the tension between neoliberal policies and community psychology unfolds, the ways in which the community perspective is integrated into a set of government programs in Chile, which are declared "community", are described and analyzed. The results show that the public policy programs reviewed, do not meet the possibilities of the PC to strengthen communities. There is an urgent need to recover and strengthen the consideration of the ethical-political dimension in professional training and the participation of the PC in public policies.

\section{Keywords}

social programs, neoliberalism, community psychology

\footnotetext{
${ }^{1}$ Correspondencia al primer autor Hector Berroeta en el correo hector.berroeta@uv.cl. El estudio reportado en este artículo es parte del proyecto FONDECYT n ${ }^{\circ} 1080528$ Etica y Política ¿dimensiones olvidadas en la psicología comunitaria hoy? (2013-2015) del mismo equipo de investigación que el equipo autor.
} 


\section{COMMUNITY PSYCHOLOGY, SOCIAL PROGRAMS AND NEOLIBERALISM: THE CHILEAN EXPERIENCE}

La Psicología Comunitaria (en adelante PC), como disciplina teórico-práctica, enfatiza la promoción del bienestar comunitario como un objetivo fundamental para el desarrollo de las sociedades postindustriales (Francescato, Tomai \& Mebane, 2006). Sin embargo, diversas investigaciones refieren que el desarrollo de políticas sociales gestadas en un sistema neoliberal, inciden negativamente en la organización comunitaria (Brady, Schoeneman \& Sawyer, 2014; Hasenfeld \& Garrow, 2012). La influencia del neoliberalismo en el desarrollo de un cierto tipo de política pública afecta fuertemente las prácticas comunitarias a la base de los programas que se implementen.

En este sentido Chile es un caso de aplicación paradigmática del modelo neoliberal, ya que se ha constituido, en una especie de bastión donde se han ensayado políticas neoliberales que, más tarde, se implementan en el resto de los países de la región a través de la participación de agencias internacionales como el Banco Interamericano de Desarrollo (BID); habiéndose reportado que los fundamentos y prácticas implementadas en los programas sociales de corte comunitario presentes en la oferta pública, se caracterizan por tener una escasa convergencia con los principios y valores ético-políticos de la PC (Reyes, Olivares, Berroeta \& Winkler, 2016).

Con el objetivo de conocer el modo en que se despliega la tensión entre políticas neoliberales y psicología comunitaria, nos propusimos describir y analizar las formas en que se integra la perspectiva comunitaria en un conjunto de programas gubernamentales en Chile que se declaran "comunitarios" en tanto, estos son los espacios donde se despliega el ejercicio profesional de la disciplina y se concretan sus aportes teórico-prácticos. La interrogante mayor a la que buscamos contribuir es ¿Es posible ejecutar programas sociales que respeten y fortalezcan las comunidades en un contexto de políticas neoliberales?

\section{Contexto neoliberal, políticas públicas y PC en Chile}

El profundo cambio cultural del que estamos siendo testigos en las últimas décadas se constituye en uno de los efectos principales de la consolidación del neoliberalismo como modelo civilizatorio a nivel global (Harvey, 2005). Según Laval y Dardot (2013, p. 15) "el neoliberalismo se puede definir como el conjunto de los discursos, de las prácticas, de los dispositivos que determinan un nuevo modo de gobierno de los hombres según el principio universal de la competencia".

Diversas investigaciones muestran que el debilitamiento de los estados de bienestar contemporáneos ha tenido como consecuencia que las comunidades deban compensar la ineficiencia de los sistemas de protección y servicios públicos, creando dispositivos que les permitan crear condiciones para el desarrollo y bienestar ciudadano (Harvey, 2005; Prasad, 2006; Schram, Soss, Houser \& Fording, 2010; Hasenfeld \& Garrow, 2012; De la Maza, 2011; Brady, Schoeneman \& Sawyer, 2014; Ortiz, 2014; Fuenmayor, 2014). Específicamente, se han identificado contradicciones entre las propuestas de organización e intervención comunitarias y la formulación de políticas sociales ancladas en sistemas político-económicos neoliberales.

La gestión pública basada en la privatización y descentralización de los servicios de salud y sociales ha repercutido fuertemente en la sociedad civil, reduciendo su capacidad crítica y cuestionadora del Estado, convirtiéndola en un cliente-consumidor más, que se victimiza y somete a los principios del mercado (Hasenfeld \& Garrow, 2012). También ha impactado en la formación y consolidación de los procesos de organización comunitaria a través de tres tendencias: (1) la promoción de una práctica basada en la evidencia como un proceso dominante para guiar la acción profesional en el tercer sector (Mulally, 2007; Rothman, 2007; Brady, 2012); (2) la atención disminuida y la tergiversación de los movimientos sociales en el contexto teórico-práctico de la educación de las comunidades (Pyles, 2009; Brady, 2012; Choudry \& Shragge, 2011; Brady, Schoeneman \& Sawyer, 2014); y (3) la creciente profesionalización de la organización comunitaria 
en el marco de los profesionales que intervienen y de sus líderes o representantes (Bar-On, 1999; Schram, Soss, Houser \& Fording, 2010; Brady, 2012).

Como consecuencia, el desarrollo y la organización comunitaria en la actualidad se ha visto fuertemente afectada por una serie de prácticas tecno-científicas, centradas en formas "objetivas" de conocimiento que se traducen en intervenciones de corte positivista/cuantitativo, que propenden al status quo a través de la promoción de reformas menores y fortalecen el individualismo neoliberal (Brady, Schoeneman \& Sawyer, 2014; Schram, Soss, Houser \& Fording, 2010).

En otros países se ha documentado que el aumento de los proyectos de intervención comunitaria no tiene un impacto directo en el fortalecimiento del compromiso político de los/as psicólogos/as sociales, ni ha aumentado la participación de las comunidades involucradas, como describe Fátima Quintal de Freitas (2000) para Brasil. Maritza Montero (2010) identifica como uno de los principales focos de tensión la verticalidad de la relación que establece la política pública con las comunidades en Venezuela. Muchos de los programas públicos son dirigidos desde afuera hacia adentro, o de arriba hacia abajo, sin informar, ni sensibilizar a la comunidad respecto de las soluciones o cambios propuestos.

En este sentido, la metáfora de haber "bailado con un monstruo", acuñada por Marybeth Shinn (2007), sintetiza bien la complejidad que describe Montero (2010) para llevar a cabo una intervención comunitaria en el contexto de programas sociales o como parte de las políticas públicas. Sin embargo, a pesar de estas dificultades, la literatura reciente plantea como deseable la contribución mutua entre estos campos (Montero, 2009; Ornelas, Vargas \& Duarte, 2010; Rodríguez, 2012; Nelson, 2013; Alfaro, 2013, Wiesenfeld, 2014).

Nelson (2013) señala que las políticas desarrolladas en Estados neoliberales, en general, enfatizan valores que serían contrarios al empoderamiento colectivo y la justicia social, tales como el individualismo, la competencia y la dependencia en uno mismo y en el mercado más que en el Estado. Para el caso de Latinoamérica la hegemonía del modelo neoliberal ha generado, según Rodríguez (2012), "fenómenos de creciente pobreza y exclusión social, de fragmentación y debilitamiento de las redes sociales y la preponderancia del individualismo sobre los valores colectivos y solidarios" (p. 133).

\section{Caso Chileno}

En Chile, según Esping-Andersen, Katzman y Wormald (2002), no existen dudas que el modelo social vigente corresponde a un régimen liberal. El modelo neoliberal que se instaló con la dictadura y consolidó con los gobiernos de la Concertación (Garretón, 2013) disminuyó el rol del Estado, condicionándolo a una función subsidiaria, liberalizó el mercado en el marco de una economía orientada a la exportación, el comercio internacional y al mercado de capitales, transformando el mercado de trabajo, los principios de protección social y los servicios públicos (Arteaga \& Martuccelli, 2012). Es así que se flexibilizó el mercado laboral, descentralizó la negociación colectiva, privatizaron las pensiones y aumentó significativamente el peso del mercado en la provisión del bienestar social (Riesco, 2009). El neoliberalismo, en definitiva, produjo una marcada desigualdad estructural y generó polos de acumulación y concentración de la riqueza, que han resultado muy difíciles de desmontar (Garretón, 2007, 2013). En política social se disminuyeron los principios de cobertura universalistas, en favor de programas específicos dirigidos a la superación de la pobreza y diseñados para no inducir dependencia asistencialista (IPPE-UDP, 2009; Molina, 2006).

Desde la recuperación de la democracia, se aprecia una reactivación de las políticas públicas dando prioridad al área social, la que se expresa inicialmente en una fuerte inversión en infraestructura social básica en las áreas de educación, salud, vivienda y obras públicas, para luego aplicarla en fórmulas que apuestan a un crecimiento con equidad (De la Maza, 2004). En este 
sentido, la inversión social se asume creando centros de atención ideados como instrumentos económicos, científicos y humanos con el fin de potenciar, paradójicamente, condiciones de libertad, igualdad y del progreso socio-económico de los ciudadanos (Krause \& Jaramillo, 1998; Rozas, 1995; 2006; Alfaro, 2007). En este marco, las intervenciones sociales se estructuran en forma de "proyectos" que requieren demostrar sus sustentabilidad e impacto (Alfaro, 2007; Reyes, 2007), optando por una estrategia de focalización selectiva dirigida a los denominados "grupos vulnerables" (etnias, mujeres, personas con discapacidades, infancia, juventud, tercera edad, entre otras) y creando instituciones al servicio de la intervención en procesos psicosociales ligados a la subjetividad y sociabilidad (Alfaro \& Zambrano, 2009).

De la Maza (2004) subraya que las políticas sociales en Chile no se han orientado al fortalecimiento de la sociedad civil, pues los espacios de participación que se han abierto a las organizaciones sociales se dirigen desde una lógica de co-financiamiento público-privado de los programas, como modo de subsidiar los insuficientes recursos del sector público. Indudablemente, este es un factor relevante a la hora de considerar la implementación de una política pública basada en una modalidad de "externalización de servicios" y en una co-participación ciudadana que se concreta en formas de capacitación laboral y fomento productivo (De la Maza, 2004). Es por ello que probablemente muchos de los programas y proyectos sociales que se implementan actualmente en Chile, se traducen en acciones que instrumentalizan la participación de la ciudadanía, sin lograr la construcción de una asociatividad de tipo permanente, siendo más bien fragmentada y efímera (De la Maza, 2004).

En este contexto de política pública altamente focalizada y con énfasis en el desarrollo individual, el enfoque comunitario tiene una escasa presencia (Reyes, Olivares, Berroeta \& Winkler, 2015) que dificulta el quehacer profesional respecto de la calidad de las intervenciones comunitarias, "situando a los/as psicólogos/as como técnicos, en un entorno laboral precario e inespecífico" (p. 388), declarando objetivos comunitarios, mientras se presenta un enfoque asistencialista e individualista.

A pesar de ello, se coincide en señalar que la expansión y consolidación de la PC chilena, producida en las últimas dos décadas, se encuentra estrechamente vinculada a la expansión de políticas públicas en el país (Alfaro \& Zambrano, 2009; Berroeta, Hatibovic \& Asún, 2012; Alfaro, 2013; Berroeta, 2014). El modelo neoliberal chileno se constituye en la impronta que delimita y, en algún sentido, sostiene las prácticas de la PC en el contexto actual (Reyes, Olivares, Berroeta \& Winkler, 2015).

Distintos autores señalan que la relación entre PC y políticas sociales en Chile se ha traducido en un enfoque individual para el abordaje de las problemáticas sociales (Alfaro, 2007; Berroeta, 2011; Berroeta et al, 2012). En dicho contexto, Berroeta (2011) plantea que se le asigna "poco valor a las condicionantes sociales, a los recursos comunitarios y a la participación ciudadana" (p. 41); tal enfoque individual, relegaría a un segundo plano las iniciativas territoriales y estaría relacionado con la distancia que adoptó la disciplina respecto de sus propios principios transformadores a partir del fuerte proceso de institucionalización experimentado (Berroeta et al., 2012).

Como reportan Krause, Jaramillo, Monreal, Carvacho y Torres (2011), la PC chilena desarrolla modelos y técnicas en distintos dispositivos de intervención públicos: asociatividad y apoyo psicosocial en el Fondo de Solidaridad e Inversión Social [FOSIS]; intervención en crisis y promoción de redes en el Servicio Nacional de Menores [SENAME]; autogestión y participación en el Ministerio de Vivienda Urbana [MINVU], promoción e inserción social en los programas de salud mental o empowerment en los programas de seguridad pública.

En suma, si bien la incorporación de la PC al campo de las políticas sociales ha favorecido su consolidación profesional y académica, los escenarios y condiciones del modelo neoliberal generan retos para la producción de prácticas comunitarias que promuevan el bienestar no solo individual sino también colectivo, por tanto es relevante revisar el estado de estas prácticas en aquellos programas sociales que son la máxima expresión del enfoque comunitario en la actual 
política social chilena.

\section{Psicología comunitaria en la práxis de las políticas públicas: desde el asistencialismo a la transformación}

La PC se ha propuesto desde sus inicios la promoción del bienestar y el desarrollo de estrategias para que las comunidades forjen su propio destino. En términos más específicos, Wiesenfeld (2014) plantea que la PC trabaja mediante procesos psicosociales vinculados con la calidad de vida de la comunidades en desventaja social, fortaleciendo sus competencias, con el propósito de conseguir recursos necesarios que les permitan ejercer su ciudadanía en condiciones de justicia y equidad.

A la base del trabajo comunitario existen distintas estrategias, como la reflexión fundamentada en el diálogo, que supone un análisis crítico que permite la desnaturalización de relaciones de poder (Nelson \& Prilleltensky, 2010) en espacios de agencialidad donde hay cabida para la transformación social, concientización y participación comunitaria. Según Montero (2004a), junto a estos procesos existen principios que organizan valóricamente lo que se hace y cómo se hace aquello que se hace. Hay dos principios que se destacan y que se relacionan con dos ámbitos distintos pero complementarios: los valores vinculados al hacer, por un lado, y los vinculados al ser, por otro. Entre los primeros, destacan como principios rectores la idea de mínimo necesario versus el máximo deseable y la devolución sistemática del conocimiento producido. Ambos son claves en el desarrollo de intervenciones que sientan sus bases en los fundamentos de la PC.

En relación a los valores referidos al ser, se destaca: la noción de las participantes como sujetas sociales, con capacidad de decisión y transformación; el valor de la igualdad, ligado a la libertad y la transformación social; y por último, el valor de la conciencia y la capacidad de problematizar la realidad (Montero, 2004a; Nelson \& Prilleltensky, 2010).

Respecto a la función de los valores, en el caso de América Latina, la situación es crítica debido a las limitantes existentes en los modos en que se proponen las intervenciones desde las políticas sociales, pues las comunidades pocas veces son consideradas en la definición de los objetivos principales (Martínez, Jaramillo, Santelices \& Krause, 2009).

Al respecto, Prilleltensky (2001) señala que es poco común que las políticas incorporen la participación de las comunidades, al menos no como es conceptualizada por la PC. Por el contrario, como revela la crítica de límites (Burton \& Kagan, 2003), los discursos políticos dominantes limitan la participación de usuarios en procesos de evaluación y diseño, de forma que la mayoría son concebidas en ausencia de sus afectados (Burton \& Kagan, 2003; Prilleltensky, 2001). Por lo demás, según sostiene Giorgi (2012), las planificaciones pseudo-participativas en que se excluye a quienes se ven afectados resultarían "incompatibles con (...) el fortalecimiento de los sujetos colectivos, el respeto a sus capacidades organizativas y los procesos de autonomía y emancipación" (p. 201).

Prilleltensky (2004) nos señala que existen muchas intervenciones en el campo de la PC, que por bien intencionadas que sean, no alteran las estructuras sociales, pues están más bien dirigidas a ayudar a los sujetos, que a modificar estructuralmente sus condiciones de vida. A partir de esta evidencia, propone la noción de validez psicopolítica como un modelo de guía valórica, que integra la orientación del campo más dirigida al mejoramiento y los desarrollos más encaminados a la transformación. De esta forma, plantea la necesidad de que la PC y las ciencias sociales pongan al centro de su reflexión, la conciencia del rol que juega el poder en el bienestar, la opresión y la justicia en los dominios personal, relacional y colectivo, como una forma de reducir la distancia entre teoría y práctica. Dicho marco nos ha servido de inspiración y contexto para estudiar tres programas de políticas sociales, intentando cubrir los espacios de representación en que se despliegan dichos programas de intervención comunitarios, siguiendo específicamente la propuesta de siete dimensiones que caracterizan la intervención comunitaria como asistencialista o 
transformadora, desarrollada por Nelson y Prilleltensky (2010).

\section{Método}

Nos aproximamos a la oferta de programas públicos vigentes en el contexto gubernamental chileno, a partir de la estructuración de un dispositivo de investigación inspirado en los principios de la teoría fundada empíricamente (Glaser \& Strauss, 1967), organizado en dos fases de estudio consecutivas. En una primera fase, a partir de un proceso secuencial de revisión, se identificó -en toda la oferta programática del Estado-, aquellos programas sociales de orientación comunitaria definidos a partir de ciertos criterios teóricos. Se revisaron 23 ministerios y 101 programas, de los cuales sólo 9 cumplían con los siguientes criterios: que estuviese orientado a la comunidad, operase a nivel territorial y tuviese vinculación en contexto comunitario. Una vez revisados y fichados, consultando en las páginas web de los respectivos ministerios y en documentos de acceso público, agregamos otros dos criterios (al menos 6 meses de duración y vigentes desde el año 2010); y sólo 5 de ellos realmente cumplían con éstos, para ser considerados potenciales estudios de casos. Elegimos un programa por ministerio, consideramos la accesibilidad de los contactos y que fueran ejecutados tanto en la Región Metropolitana como en la Quinta Región, con lo cual se seleccionó 3 programas que, declaran en sus bases, adscribir al enfoque comunitario y cumplían con todos los criterios de revisión: 1. Programa de Mejoramiento de la Infancia (PMI, Ministerio de Educación) 2. Programa de Prevención Comunitaria (PPC, Ministerio de Justicia) y 3) Recuperación de Barrios (Ministerio de Vivienda).

Así, en una segunda fase del estudio, se recurrió a la metodología del estudio de caso para analizar en profundidad cada uno de estos tres programas. Para acceder al discurso de los actores y procesos, realizamos entrevistas semi-estructuradas a un conjunto de diseñadores/as, ejecutores/as y usuarios/as de los programas, cuyo propósito fue conocer motivaciones, creencias, esquemas de interpretación y modos de significación que los/as entrevistados/as tenían respecto al sentido y alcance de su participación como agentes de cambio en sus diversos campos y contextos (Gaínza, 2006). Los temas de entrevista giraron en torno a distintos ejes. Para el caso de los diseñadores se preguntó por su trayectoria en el diseño de programas, por los valores y el concepto de comunidad a la base de los diseños propuestos, por el rol de comunidad, sus capacidades, recursos y ejercicio de poder. En cuanto a los ejecutores, las preguntas exploraron ámbitos como la experiencia de ejecución, rescatando fortalezas, aciertos, dificultades, nudos críticos, aprendizajes, tipo de financiamiento, rol de la comunidad, sus capacidades, recursos y ejercicio de poder. Finalmente, las preguntas a los usuarios, se concentraron fundamentalmente en la experiencia de participación, destacando procesos de convocatoria, acceso, conocimiento sobre el carácter y naturaleza del programa, sobre aprendizajes y fortalezas, debilidades y logros que se derivan de la experiencia de participación. Además, utilizamos técnicas documentales y textuales para analizar documentos, libros, comunicaciones, páginas web, como productos habituales de los distintos contextos materiales que las producen (Iñiguez, 1999). Se realizó un total de 24 entrevistas (21 individuales y 3 grupales) y se revisaron 14 documentos técnicos. 
Tabla $\mathbf{n}^{0} 1$

Descripción de participantes y documentos revisados para los programas PMI, PPC y RB

\begin{tabular}{|c|c|c|}
\hline & Participantes & Documentos \\
\hline PMI & $\begin{array}{l}3 \text { Diseñadores/as } \\
2 \text { Ejecutores/as } \\
3 \text { Usuarios/as }\end{array}$ & $\begin{array}{l}\text { 1. Evaluación PMI (2001). UAH-CIDE } \\
\text { 2. Bases Técnicas del Programa (2008): Guía Construyendo Nuestro PMI } \\
\text { 3. Evaluación PMI (2012). EPG } \\
\text { 4. Organización de Estados Iberoamericanos, OEI (s/f) Chile. Educación } \\
\text { Inicial. Experiencias no Escolarizadas. }\end{array}$ \\
\hline PPC & $\begin{array}{l}2 \text { Diseñadores/as } \\
4 \text { Ejecutores/as } \\
12 \text { Usuarios/as }\end{array}$ & $\begin{array}{l}\text { 1. Ley número } 20.032 \text { (2005) } \\
\text { 2. Orientaciones Técnicas Centros Comunitarios por los Derechos Infanto } \\
\text { Juveniles/CCIJ (2004) } \\
\text { 3. Resumen ejecutivo estudio: "Generando un modelo para la alerta } \\
\text { temprana de vulneraciones de derechos de niños, niñas y adolescentes en el } \\
\text { espacio local" (2004) } \\
\text { 4. Documento Énfasis Programáticos para los Programas de Prevención } \\
\text { Comunitaria (PPC) período 2011-2014. (2011) } \\
\text { 5. Resumen ejecutivo "Estudio de caso: Efectividad de la Labor Preventiva } \\
\text { de los Programas de Prevención Comunitaria (PPC)" (2011) }\end{array}$ \\
\hline $\mathrm{RB}$ & $\begin{array}{l}1 \text { Diseñador/a } \\
2 \text { Ejecutores/as } \\
1 \text { Usuario/a }\end{array}$ & $\begin{array}{l}\text { 1. Trabajo FASE 1: Programa de Recuperación de Barrios. MINVU } \\
\text { 2. Recuperación de } 200 \text { Barrios ( } 2010) \\
\text { 3. Decreto Supremo N } 14 \text {, (V. Y U.), De } 2007 \text { D.O. De } 12.04 .074 . \\
\text { Reglamenta Programa De Recuperación De Barrios. } \\
\text { 5. El desafío de la formulación de estrategias de intervención social en } \\
\text { barrios }\end{array}$ \\
\hline Total & 30 & 14 \\
\hline
\end{tabular}

Finalmente, los resultados se analizaron en base a la propuesta de Nelson y Prilleltensky (2010), que describe las intervenciones comunitarias como asistencialistas o transformadoras según las características que adoptan en siete dimensiones específicas: marco de temas y problemas, valores, nivel de análisis, foco en la prevención, resultados esperados, proceso de intervención, y rol de los psicólogos comunitarios.

Todas las personas participantes en el estudio aceptaron integrarse voluntariamente luego de trabajar con ellas el proceso de consentimiento informado. Esta investigación fue sometida a evaluación por parte del Comité de Ética de la Unviersidad de Santiago de Chile, siendo aprobada sin objeciones.

\section{Resultados}

Los resultados descriptivos de cada programa se presentan en el cuadro $\mathrm{N}^{\mathrm{o}} 1$, organizados en torno a siete dimensiones propuestas por Nelson y Prilleltensky (2010) elaboradas con el fin de caracterizar las intervenciones comunitarias que se desarrollan en la actualidad, mencionadas previamente. 
Cuadro n ${ }^{\circ} 1$

Análisis de programas por dimensiones.

\begin{tabular}{|c|c|c|c|c|c|}
\hline $\begin{array}{c}\text { Carac } \\
\text { terísti } \\
\text { cas }\end{array}$ & Asistencialista & Transformadora & PMI & PPC & Quiero mi Barrio \\
\hline 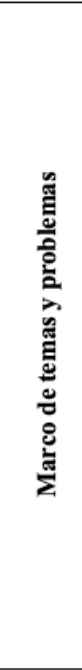 & $\begin{array}{l}\text { Los temas y } \\
\text { problemas son } \\
\text { conceptualizados } \\
\text { como materias } \\
\text { técnicas que } \\
\text { pueden ser } \\
\text { resueltas a través } \\
\text { de una resolución } \\
\text { de problemas } \\
\text { racional-empírica; } \\
\text { las dinámicas de } \\
\text { poder son } \\
\text { ignoradas. La } \\
\text { resolución } \\
\text { científica de } \\
\text { problemas está } \\
\text { como foco; el } \\
\text { poder está en el } \\
\text { fondo. }\end{array}$ & $\begin{array}{l}\text { Los temas y } \\
\text { problemas están } \\
\text { conceptualizados en } \\
\text { términos de opresión } \\
\text { e inequidades en el } \\
\text { poder que requieren } \\
\text { soluciones } \\
\text { liberadoras, así como } \\
\text { también la } \\
\text { investigación y } \\
\text { resolución de } \\
\text { problemas. } \\
\text { Poder, opresión y } \\
\text { liberación comparten } \\
\text { el foco o figura con } \\
\text { una resolución } \\
\text { científica de } \\
\text { problemas. }\end{array}$ & $\begin{array}{l}\text { El origen del programa responde a } \\
\text { una racionalidad técnica, se } \\
\text { propone como una alternativa } \\
\text { para aumentar la cobertura de } \\
\text { educación preescolar. } \\
\text { El programa propone el cuidado y } \\
\text { desarrollo de los niños desde una } \\
\text { estrategia comunitaria. Las } \\
\text { familias y la comunidad se } \\
\text { responsabilizan de su desarrollo, a } \\
\text { partir de los recursos y realidades } \\
\text { propias. } \\
\text { Técnicamente para la } \\
\text { construcción de la propuesta se } \\
\text { trabaja en equipo con solidaridad. } \\
\text { Todos pueden ser potenciales } \\
\text { educadores, el grupo decide } \\
\text { cómo, cuándo y dónde se ejecuta. }\end{array}$ & $\begin{array}{l}\text { Prevalece una mirada técnica } \\
\text { que prescribe una forma de } \\
\text { afrontar la vulnerabilidad de } \\
\text { niños y niñas, proponiendo una } \\
\text { fórmula que disminuye los } \\
\text { riesgos de tal condición (se } \\
\text { trata de una lectura técnica y } \\
\text { no política). } \\
\text { Desde el polo transformador: } \\
\text { es precisamente la dimensión } \\
\text { de poder la que permite } \\
\text { comprender el rol que juegan } \\
\text { niños y niñas en los procesos } \\
\text { de participación que } \\
\text { desencadenan } \\
\text { transformaciones en su } \\
\text { entorno. Los niños en conjunto } \\
\text { con la comunidad, } \\
\text { protagonizan los procesos de } \\
\text { cambio propuestos. }\end{array}$ & $\begin{array}{l}\text { Se define el problema } \\
\text { desde la vulnerabilidad } \\
\text { social y urbana de los } \\
\text { barrios, las } \\
\text { comunidades son } \\
\text { seleccionadas en base } \\
\text { a criterios de pobreza } \\
\text { y exclusión territorial. } \\
\text { La estrategia del } \\
\text { programa es } \\
\text { participativa; se busca } \\
\text { que los vecinos sean } \\
\text { protagonistas; se } \\
\text { favorece la } \\
\text { participación de los } \\
\text { diversos } \\
\text { representantes tanto en } \\
\text { el diagnóstico como en } \\
\text { toma de decisiones. } \\
\text { Las municipalidades y } \\
\text { comunidades son } \\
\text { actores relevantes. }\end{array}$ \\
\hline
\end{tabular}

\begin{tabular}{|c|c|c|c|c|c|}
\hline 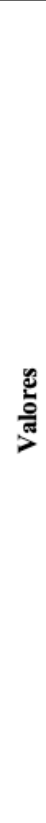 & $\begin{array}{l}\text { Debido a que los } \\
\text { temas y } \\
\text { problemas están } \\
\text { conceptualizados } \\
\text { en términos } \\
\text { técnicos, a } \\
\text { menudo se ignora } \\
\text { el énfasis en los } \\
\text { valores de la } \\
\text { intervención. Sin } \\
\text { embargo, a } \\
\text { valores como } \\
\text { holismo, salud, } \\
\text { cuidado y } \\
\text { compasión se les } \\
\text { da implícitamente } \\
\text { más énfasis. Los } \\
\text { valores están } \\
\text { como fondo. }\end{array}$ & $\begin{array}{l}\text { Los valores juegan } \\
\text { un rol central en la } \\
\text { conceptualización de } \\
\text { la intervención. } \\
\text { Aunque los valores } \\
\text { de holismo, salud, } \\
\text { cuidado y compasión } \\
\text { pueden estar } \\
\text { presentes, se pone } \\
\text { más énfasis en los } \\
\text { valores de } \\
\text { autodeterminación, } \\
\text { participación, justicia } \\
\text { social, respeto por la } \\
\text { diversidad y } \\
\text { rendición de cuentas } \\
\text { (accountability) de } \\
\text { los grupos } \\
\text { oprimidos. Los } \\
\text { valores están como } \\
\text { figura o foco. }\end{array}$ & $\begin{array}{l}\text { Este programa tiene profundos } \\
\text { fundamentos valóricos. El } \\
\text { objetivo es que los niños y niñas } \\
\text { aprendan, se desarrollen y tengan } \\
\text { un espacio donde convivir } \\
\text { sanamente. } \\
\text { A la vez, se busca que todas las } \\
\text { personas que participan en él } \\
\text { aprendan a trabajar en equipo } \\
\text { como agentes educativos y } \\
\text { compartan con solidaridad, } \\
\text { respeto y tolerancia. El principio } \\
\text { ético central corresponde al } \\
\text { respeto por el Otro y se traduce, } \\
\text { también, en la valoración de la } \\
\text { autonomía, tanto personal como } \\
\text { comunitaria. La comunidad es } \\
\text { vista como Otro válido con } \\
\text { capacidades para asumir su } \\
\text { desarrollo. Se valida el apoyo } \\
\text { mutuo y contar con el Otro en el } \\
\text { abordaje de los problemas. El } \\
\text { principal componente de la } \\
\text { relación de sus integrantes con la } \\
\text { organización refiere a intereses } \\
\text { comunes, las responsabilidades } \\
\text { son asumidas y compartidas. }\end{array}$ & $\begin{array}{l}\text { En este programa los valores } \\
\text { que se encuentran } \\
\text { implícitamente a la base de su } \\
\text { diseño se relacionan con la } \\
\text { idea de cuidado y compasión, } \\
\text { pues se asume que los niños y } \\
\text { niñas son sujetos vulnerables } \\
\text { que requieren de una } \\
\text { intervención que mejore sus } \\
\text { condiciones de vida. Esto se } \\
\text { vuelve relevante pues el } \\
\text { programa sólo se implementa } \\
\text { en aquellos sectores } \\
\text { considerados vulnerables } \\
\text { debido a sus numerosos } \\
\text { factores de riesgo. } \\
\text { Sin embargo, en el polo } \\
\text { transformador, el programa } \\
\text { también declara dentro de su } \\
\text { diseño, un marcado énfasis en } \\
\text { la promoción de la } \\
\text { participación infantil, que haga } \\
\text { posible que niños y niñas } \\
\text { formen parte de espacios } \\
\text { decisionales habitualmente } \\
\text { reservados para el mundo } \\
\text { adulto. }\end{array}$ & $\begin{array}{l}\text { El programa se } \\
\text { propone contribuir al } \\
\text { mejoramiento de la } \\
\text { calidad de vida de los } \\
\text { habitantes de barrios } \\
\text { que presentan } \\
\text { problemas de deterioro } \\
\text { urbano y } \\
\text { vulnerabilidad social, } \\
\text { a través de } \\
\text { intervenciones } \\
\text { integrales que mejoren } \\
\text { el entorno urbano y } \\
\text { promuevan el } \\
\text { fortalecimiento de la } \\
\text { participación social. } \\
\text { Como valores están el } \\
\text { respeto al medio } \\
\text { ambiente, la } \\
\text { valorización del } \\
\text { patrimonio, la } \\
\text { valoración de la } \\
\text { participación y } \\
\text { fortalecer orgullo e } \\
\text { identidad barrial. }\end{array}$ \\
\hline
\end{tabular}




\begin{tabular}{|c|c|c|c|c|}
\hline & & \multicolumn{3}{|c|}{$\begin{array}{r}\text { Revista Interamericana de Psicologia/Interamerican Journal of } \\
\text { Psychology (IJP) } \\
\text { 2019, Vol., 53, No.2, pp. 281-297 }\end{array}$} \\
\hline $\begin{array}{l}\text { Los temas y } \\
\text { problemas son } \\
\text { examinados en } \\
\text { términos de una } \\
\text { perspectiva } \\
\text { ecológica que } \\
\text { considera } \\
\text { múltiples niveles } \\
\text { de análisis. Sin } \\
\text { embargo, las } \\
\text { intervenciones } \\
\text { están a menudo } \\
\text { dirigidas a } \\
\text { mejorar el } \\
\text { bienestar personal } \\
\text { y relacional. Las } \\
\text { intervenciones a } \\
\text { nivel relacional } \\
\text { son el foco. }\end{array}$ & $\begin{array}{l}\text { Temas y problemas } \\
\text { son examinados en } \\
\text { términos de } \\
\text { dinámicas de poder } \\
\text { que son } \\
\text { conceptualizadas } \\
\text { como ocurriendo en } \\
\text { múltiples niveles de } \\
\text { análisis. La } \\
\text { intervención ocurre } \\
\text { en todos los niveles } \\
\text { de análisis, pero } \\
\text { existe un esfuerzo } \\
\text { concertado en } \\
\text { mejorar el bienestar } \\
\text { colectivo. El nivel de } \\
\text { análisis colectivo es } \\
\text { la figura incluso en } \\
\text { intervenciones a } \\
\text { nivel individual y } \\
\text { relacional. }\end{array}$ & $\begin{array}{l}\text { El programa está dirigido a niños } \\
\text { y niñas menores de seis años de } \\
\text { edad, que en su barrio o sector no } \\
\text { cuentan con acceso a la oferta de } \\
\text { educación preescolar, que viven } \\
\text { en condición de vulnerabilidad y } \\
\text { cuyas madres desean estudiar, } \\
\text { buscan trabajo, trabajan y sus } \\
\text { familias presentan indicadores de } \\
\text { vulnerabilidad para el desarrollo y } \\
\text { educación de los niños y niñas. } \\
\text { Se promueve que la familia } \\
\text { participe activamente en el } \\
\text { cuidado y la educación de sus } \\
\text { hijos a través del voluntariado de } \\
\text { madres, abuelas, tías o vecinas } \\
\text { que han trabajado con niños/as o } \\
\text { tienen interés y tiempo para } \\
\text { hacerlo. } \\
\text { El poder se pone en juego al } \\
\text { momento de comprender la } \\
\text { participación de los distintos } \\
\text { actores en el programa; lo que } \\
\text { deriva en el reconocimiento de su } \\
\text { capacidad para opinar y decidir } \\
\text { sobre los asuntos que afectan el } \\
\text { desarrollo del PMI. Es la } \\
\text { comunidad la responsable y la que } \\
\text { decide qué se hace, cómo } \\
\text { organizar y cómo definir las } \\
\text { prioridades (problemas y } \\
\text { soluciones). }\end{array}$ & $\begin{array}{l}\text { En términos asistenciales se } \\
\text { asume que los niños y niñas } \\
\text { requieren de un proceso de } \\
\text { formación que los convierta en } \\
\text { líderes de su comunidad, para } \\
\text { ello, las experiencias revisadas } \\
\text { han optado por seleccionar a } \\
\text { aquellos niños y niñas que } \\
\text { presentan mejores } \\
\text { capacidades. Esto tiene un } \\
\text { claro foco de abordaje } \\
\text { individual, pues se visibiliza al } \\
\text { líder por sobre el resto de sus } \\
\text { pares (el que se denomina } \\
\text { vocero o representante). } \\
\text { Con todo, también en su } \\
\text { diseño, el programa contempla } \\
\text { una lectura sobre la dimensión } \\
\text { de poder, que explica las } \\
\text { desigualdades entre adultos y } \\
\text { niños y lo conceptualiza como } \\
\text { "adultocentrismo". Este tema } \\
\text { es abordado como eje de } \\
\text { trabajo, en las experiencias de } \\
\text { ejecución analizadas. }\end{array}$ & $\begin{array}{l}\text { Los objetivos del } \\
\text { programa están } \\
\text { centrados en múltiples } \\
\text { niveles de análisis, se } \\
\text { promueve el } \\
\text { mejoramiento y la } \\
\text { dotación sostenible de } \\
\text { equipamientos, } \\
\text { infraestructura y } \\
\text { espacios públicos de } \\
\text { los barrios, orientados } \\
\text { a las necesidades de } \\
\text { sus habitantes y la } \\
\text { integración territorial. } \\
\text { Se busca el bienestar } \\
\text { colectivo, integrando } \\
\text { políticas públicas con } \\
\text { intereses de la } \\
\text { comunidad y aportes } \\
\text { del mundo privado. }\end{array}$ \\
\hline
\end{tabular}

\begin{tabular}{|c|c|c|c|c|c|}
\hline 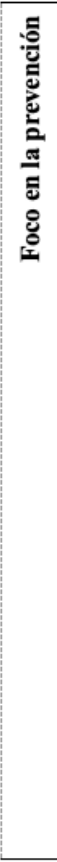 & $\begin{array}{l}\text { La prevención es } \\
\text { buscada } \\
\text { primariamente } \\
\text { como mejoría de } \\
\text { los factores } \\
\text { protectores, } \\
\text { incluyendo } \\
\text { habilidades, } \\
\text { autoestima y } \\
\text { sistemas de } \\
\text { apoyo. }\end{array}$ & $\begin{array}{l}\text { La prevención es } \\
\text { buscada } \\
\text { primariamente en la } \\
\text { prevención de } \\
\text { factores sistemáticos } \\
\text { de riesgos, } \\
\text { incluyendo racismo, } \\
\text { sexismo y pobreza }\end{array}$ & $\begin{array}{l}\text { Se aprecia un foco de prevención } \\
\text { en la línea asistencialista que pone } \\
\text { énfasis en factores protectores, } \\
\text { como educación y familia. La } \\
\text { prevención también se aborda } \\
\text { desde una dimensión macrosocial, } \\
\text { pues se asume que el programa es } \\
\text { una estrategia que previene la } \\
\text { vulnerabilidad en contextos de } \\
\text { pobreza. Desde una perspectiva } \\
\text { de Derechos Humanos se asume } \\
\text { que la intervención favorece la } \\
\text { redistribución de poder al interior } \\
\text { de las comunidades, estableciendo } \\
\text { relaciones horizontales al interior } \\
\text { de la sociedad. }\end{array}$ & $\begin{array}{l}\text { El programa, es marcadamente } \\
\text { asistencialista, pues declara } \\
\text { como horizonte la prevención } \\
\text { a través del fortalecimiento de } \\
\text { factores protectores. En este } \\
\text { sentido, propone a la } \\
\text { participación infantil como un } \\
\text { factor protector ante el } \\
\text { contexto de vulnerabilidad en } \\
\text { que se encuentran los niños y } \\
\text { niñas. } \\
\text { De todos modos, nomina la } \\
\text { prevención como de tipo } \\
\text { comunitaria, entendiendo su } \\
\text { alcance en un nivel relacional. }\end{array}$ & $\begin{array}{l}\text { La intervención en el } \\
\text { barrio promueve el } \\
\text { mejoramiento y la } \\
\text { revitalización tanto de } \\
\text { los espacios públicos } \\
\text { colectivos como de la } \\
\text { red social de los } \\
\text { barrios, enfrentando } \\
\text { problemas de deterioro } \\
\text { urbano, tales como } \\
\text { conectividad e } \\
\text { integración con el } \\
\text { resto de la ciudad, } \\
\text { déficit de } \\
\text { pavimentación, } \\
\text { ausencia o mala } \\
\text { calidad de } \\
\text { equipamiento } \\
\text { comunitario, áreas } \\
\text { verdes, iluminación, y } \\
\text { otras obras; } \\
\text { recuperando a su vez } \\
\text { la confianza y la } \\
\text { participación de los } \\
\text { vecinos en el proceso } \\
\text { de mejoramiento de su } \\
\text { entorno urbano } \\
\text { inmediato. }\end{array}$ \\
\hline
\end{tabular}




\begin{tabular}{|c|c|c|c|c|c|}
\hline 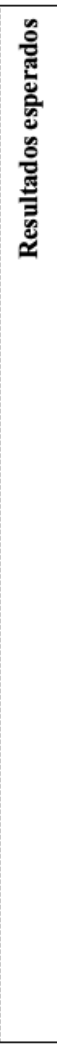 & $\begin{array}{l}\text { El primer resultado } \\
\text { esperado es } \\
\text { aumentar el } \\
\text { bienestar, que es } \\
\text { conceptualizado } \\
\text { apolíticamente y } \\
\text { en forma } \\
\text { circunscrita a nivel } \\
\text { individual de } \\
\text { análisis. } \\
\text { Resultados } \\
\text { específicos } \\
\text { incluyen: la } \\
\text { promoción del } \\
\text { bienestar } \\
\text { individual que } \\
\text { incluye } \\
\text { autoestima, } \\
\text { independencia y } \\
\text { como autonomía. } \\
\text { Considera la } \\
\text { prevención de } \\
\text { problemas } \\
\text { psicosociales en la } \\
\text { vida y el aumento } \\
\text { del apoyo social. } \\
\text { Los resultados a } \\
\text { nivel individual } \\
\text { son la figura }\end{array}$ & $\begin{array}{l}\text { El resultado deseado } \\
\text { primariamente es } \\
\text { aumentar el bienestar } \\
\text { que es } \\
\text { conceptualizado en } \\
\text { términos de poder en } \\
\text { múltiples niveles de } \\
\text { análisis. Resultados } \\
\text { esperados específicos } \\
\text { incluyen: control } \\
\text { aumentado, elección, } \\
\text { autoestima, } \\
\text { competencia, } \\
\text { independencia, } \\
\text { conciencia politica } \\
\text { derechos políticos y } \\
\text { una identidad } \\
\text { positiva; aumento en } \\
\text { las relaciones sociales } \\
\text { de apoyo y } \\
\text { participación en la } \\
\text { vida social } \\
\text { comunitaria y política; } \\
\text { la adquisición de } \\
\text { recursos valorados, } \\
\text { tales como empleo, } \\
\text { ingreso, educación y } \\
\text { vivienda; y libertad } \\
\text { del abuso, la violencia } \\
\text { y la explotación. Los } \\
\text { resultados en } \\
\text { múltiples niveles de } \\
\text { análisis que enfatizan } \\
\text { compartir el poder y } \\
\text { la equidad son la } \\
\text { figura. }\end{array}$ & $\begin{array}{l}\text { El objetivo central es el bienestar de } \\
\text { los niños y niñas, que aprendan, se } \\
\text { desarrollen y tengan un espacio de } \\
\text { convivencia. A la vez, se busca que } \\
\text { todas las personas que participan, } \\
\text { aprendan a trabajar en equipo como } \\
\text { agentes educativos y compartan con } \\
\text { solidaridad, respeto y tolerancia. } \\
\text { En la concepción de la organización } \\
\text { y de las actividades prevalece el } \\
\text { interés por fomentar un aumento } \\
\text { del poder de las propias integrantes } \\
\text { y usuarias Al mismo tiempo se } \\
\text { fomenta el Apadrinamiento, que } \\
\text { corresponde a un tipo de relación } \\
\text { basada en la beneficencia y } \\
\text { dependencia a ciertos grupos que } \\
\text { tienen más recursos, promoviendo } \\
\text { un intercambio desigual y que } \\
\text { obstaculizan el desarrollo de poder } \\
\text { propio. }\end{array}$ & $\begin{array}{l}\text { Los ejecutores y también los } \\
\text { participantes reconocen la } \\
\text { importancia de adquirir } \\
\text { experiencias de desarrollo } \\
\text { personal que fortalezcan la } \\
\text { autoestima que les permita } \\
\text { relacionarse con los adultos de } \\
\text { su comunidad desde otros } \\
\text { lugares. } \\
\text { Se destacan como resultados } \\
\text { relevantes, sobre todo en el } \\
\text { relato de los ejecutores, algunas } \\
\text { experiencias de incidencia } \\
\text { política que han permitido } \\
\text { posicionar a niños y niñas como } \\
\text { un referente político en su } \\
\text { localidad. }\end{array}$ & $\begin{array}{l}\text { Se espera resultados a } \\
\text { nivel colectivo, } \\
\text { integrales, desde el } \\
\text { mejoramiento de la } \\
\text { infraestructura de } \\
\text { barrio, hasta la } \\
\text { identidad y } \\
\text { asociatividad en la } \\
\text { comunidad. } \\
\text { Resultados concretos y } \\
\text { resultados simbólicos, } \\
\text { relacionales. El } \\
\text { bienestar individual } \\
\text { aparece supeditado al } \\
\text { bienestar colectivo. }\end{array}$ \\
\hline
\end{tabular}

\begin{tabular}{|c|c|c|c|c|c|}
\hline 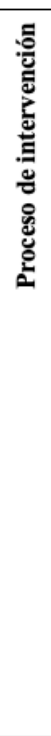 & $\begin{array}{l}\text { El proceso de } \\
\text { intervención } \\
\text { puede ser "guiado } \\
\text { por expertos" } \\
\text { pero usualmente } \\
\text { involucra la } \\
\text { colaboración con } \\
\text { múltiples } \\
\text { "stakeholders" de } \\
\text { la comunidad. }\end{array}$ & $\begin{array}{l}\text { El proceso de } \\
\text { intervención } \\
\text { involucra } \\
\text { colaboración } \\
\text { "partnership" en la } \\
\text { que psicólogos } \\
\text { comunitarios } \\
\text { trabajan } \\
\text { solidariamente con } \\
\text { los grupos oprimidos } \\
\text { y otros posibles } \\
\text { agentes } \\
\text { comunitarios. } \\
\text { Concientización, } \\
\text { compartir el poder, } \\
\text { aprendizaje mutuo, } \\
\text { resistencia, } \\
\text { participación, } \\
\text { relaciones de apoyo e } \\
\text { igualitarias y } \\
\text { movilización de } \\
\text { recursos están como } \\
\text { figura en el proceso } \\
\text { de intervención. }\end{array}$ & $\begin{array}{l}\text { No participan psicólogos/as. Se } \\
\text { define por técnicos externos a la } \\
\text { comunidad. Destaca la } \\
\text { importancia de la participación de } \\
\text { distintos actores para promover } \\
\text { espacios de co-construcción y } \\
\text { colaboración comunitaria. La } \\
\text { participación de la comunidad } \\
\text { tiene distintos grados: va desde la } \\
\text { sola asistencia de los participantes } \\
\text { o sus familias a convocatorias } \\
\text { específicas, en un grado más } \\
\text { profundo, se relaciona con la } \\
\text { posibilidad de opinar y tomar } \\
\text { decisiones sobre actividades o } \\
\text { cambios al interior del programa. }\end{array}$ & $\begin{array}{l}\text { La intervención es desarrollada } \\
\text { fundamentalmente por los } \\
\text { equipos profesionales, desde } \\
\text { un énfasis técnico. } \\
\text { La comunidad sólo colabora } \\
\text { ocasionalmente, pero no llega } \\
\text { a ser parte de la toma de } \\
\text { decisiones técnicas del } \\
\text { proceso. }\end{array}$ & $\begin{array}{l}\text { La intervención es } \\
\text { facilitada por una } \\
\text { dupla técnica, } \\
\text { compuesta por dos } \\
\text { profesionales uno del } \\
\text { área social y otro del } \\
\text { área urbana. La } \\
\text { comunidad decide los } \\
\text { proyectos de inversión } \\
\text { a través de un } \\
\text { conglomerado } \\
\text { compuesto por } \\
\text { representantes de } \\
\text { organizaciones } \\
\text { barriales. }\end{array}$ \\
\hline
\end{tabular}

Como se puede apreciar, los programas analizados conceptualizan los problemas que abordan de un modo técnico, se proponen como estrategias focalizadas que buscan mejorar la calidad de vida de los usuarios y las formas de relación al interior de la comunidad. Si bien se espera que la resolución de los problemas se alcance mediante acciones colectivas de la comunidad, no se identifica que las intervenciones pongan en el centro de su quehacer el rol que juega el poder, la opresión y la liberación. 
Por su parte, todos los programas estudiados fundamentan su existencia en valores claros y definidos. En sus diseños se promueve el autocuidado, el respeto por el Otro, la tolerancia y la participación. Sin embargo, valores que enfatizan una reflexión estructural de los cambios, como la justicia o autodeterminación, se encuentran ausentes en todos los programas analizados.

Los tres programas trabajan a nivel individual y relacional, ponen énfasis en el desarrollo de procesos participativos y formación de líderes comunitarios. El bienestar colectivo es situado como una finalidad concreta, producto de los logros concretos del programa, y no como un esfuerzo sistemático de redistribuir el poder entre los distintos niveles implicados en los problemas que se abordan.

Los programas centran la prevención en la minimización de los factores de riesgo y en la potenciación de factores protectores de las comunidades. Sin embargo, las estrategias participativas que se despliegan, buscan favorecer cambios en las formas relacionales al interior de las comunidades, de tal forma que se generen transformaciones en las dinámicas de riesgo que sustentan las condiciones de pobreza que afectan a las comunidades intervenidas.

Los resultados que se espera alcanzar en los programas analizados, están centrados en aumentar el bienestar individual y colectivo, buscando el desarrollo personal de los participantes, que mejoren las dinámicas de relación y la toma de control personal, a la vez que se espera que la promoción de acciones colectivas genere cambios que puedan traducirse en el fortalecimiento organizacional y en el aumento de la asociatividad comunitaria.

Los programas son definidos en su origen, por profesionales expertos/as externos a la comunidad. La profundidad de la participación de la comunidad en la toma de decisiones es diversa y se expresa, fundamentalmente, en la ejecución específica de actividades y en la definición de metas a alcanzar. Es posible advertir la generación de procesos de aprendizaje mutuo entre profesionales y agentes comunitarios en un marco de relaciones colaborativas.

El rol de los/as psicólogos/as comunitarios/as es indefinido. Lo que se evidencia en los programas analizados es que como profesionales no cumplen una función específica e incluso pueden no formar parte de los equipos que trabajan directamente con las comunidades. Son prescindibles para las intervenciones emprendidas; se limitan, como otros profesionales de las ciencias sociales, a ejecutar los lineamientos técnicos de los programas ya diseñados, por lo que no despliegan un rol político en su quehacer.

\section{Discusión y Conclusiones}

La implantación del modelo neoliberal en la sociedad chilena ha tenido efectos en las formas de convivencia, penetrando transversalmente ámbitos de la vida cotidiana que quedan inmersos en lógicas individualizantes, competitivas y que se alejan de los principios valóricos propuestos desde los orígenes de la PC (Nelson, 2013; Montero, 2010).

Los resultados obtenidos muestran que los programas de políticas públicas revisados, que se declaran comunitarios, no cumplen a cabalidad con las posibilidades de la PC para fortalecer las comunidades en la búsqueda de un bienestar colectivo que beneficie a amplios sectores de la sociedad. Postulamos que ello se relaciona de forma directa con el modelo neoliberal imperante, que se contrapone a los principios, objetivos y valores de la PC. Con ello, la definición de los programas como comunitarios queda a nivel de discurso y no se traduce en las prácticas ni en su implementación. De este modo, se evidencia la urgente necesidad de recuperar y fortalecer la consideración de los valores -la dimensión ética- (Nelson, 2013) y la redistribución del poder -la dimensión política- en la formación profesional y la participación de la PC en las políticas públicas.

La experiencia a nivel internacional muestra, hace más de una década, los efectos contradictorios de las políticas públicas diseñadas desde una lógica managerial y cómo, en un contexto de gobernanza, las comunidades tienden a ser instrumentalizadas. Al respecto, resulta 
relevante destacar que investigaciones recientes en Chile complementan la información reportada en este estudio, pues a partir del análisis de diferentes programas sociales se ha observado que éstos tienden a performar sujetos sociales individualistas, empresarios de sí mismos, que se muestran pasivos frente al sistema y abiertos/agradecidos frente a lógicas de beneficencia y asistencialidad (Rivera, 2017; Astete \& Vacari, 2017; Reyes, Jaramillo, Pizarro, Vergara, Navarrete \& Yáñez, en prensa).

Respecto a esto último, las políticas sociales existentes en la actualidad son claras en su objetivo y su alcance: no buscan transformar la sociedad de manera profunda, sino que, su principal propósito es generar ajustes para que aquellos sectores que se encuentran en desventaja social (los llamados "sectores vulnerables", focos de la política social) puedan integrarse a las dinámicas económicas y sociales dominantes, disminuyendo los índices de pobreza y desigualdad existentes, a través de la participación en proyectos que deben demostrar sustentabilidad y efectividad (Alfaro, 2007; Reyes, 2007). De este modo, el desarrollo de política social, cumple una función compensatoria y estabilizadora de un orden social y económico, produciendo marcos de actuación profesional que tienen un fuerte componente técnico ("el hacer") por sobre una dimensión éticopolítica ("el ser") que queda ubicada en un nivel discursivo en los diseños de la política social.

Como señalan Nelson \& Prilleltensky (2010) en el marco general dado por la política social, si bien existe una presencia mayoritaria de acciones centradas en el polo asistencialista también es posible reconocer acciones del polo transformativo. En nuestros resultados hay antecedentes que merecen ser relevados por su potencial para crear pistas que orienten futuras acciones hacia el polo transformador. A pesar de no incorporar el rol del poder en el centro de los programas, los diseños propuestos son una oportunidad para el cambio, pues constituyen un punto de partida concreto para quienes trabajamos en el nivel de la implementación. Desde estos espacios, se puede justificar el uso de estrategias participativas que avancen en lo decisional y eviten la instrumentalización de la ciudadanía. Con ello, pensamos, podemos avanzar en la construcción de una asociatividad de tipo permanente que consolide espacios democráticos para la sociedad civil (De la Maza, 2004), permitiendo impactar a nivel de la subjetividad de los participantes y en el respeto a los derechos de las comunidades ante las intervenciones sociales (Olivares, Winkler, Reyes, Berroeta \& Montero, 2018).

Si bien la mayoría de los programas siguen centrados en un nivel individual (Berroeta, 2014), promoviendo liderazgos que reproducen la fragmentación y la competitividad entre comunidades (Rodríguez, 2012), de todos modos existe una preocupación por el desarrollo comunitario, instalando la necesidad de avanzar hacia relaciones de colaboración y solidaridad donde la emergencia de un actor colectivo cobre protagonismo al interior de los programas públicos. Es así como se vuelve relevante reconocer que toda política social que se inscriba en un contexto neoliberal tiene componentes y niveles que es necesario diferenciar y que, quienes trabajamos en ese marco, debemos conocer los procesos involucrados y los niveles en que se despliega cada estrategia, asumiendo la importancia de trabajar en el dominio individual, relacional y colectivo sin privilegiar uno sobre otro (Prilleltensky, 2004).

Así, vemos que en muchos casos, la presencia de discursos y valores asociados a la transformación logran traducirse en prácticas que impactan concretamente a las comunidades permitiendo acceso al poder, tal y como lo vimos en uno de los programas analizados, en la medida que permitía avanzar hacia un horizonte transformador dando acceso a que niños y niñas tomaran decisiones sobre sus propias necesidades, fortaleciendo la propia agencia y consolidando un rol político (Olivares \& Reyes, 2016). Sin embargo, tales avances advertidos a partir de esta experiencia, no lograron consolidarse, pues luego de 10 años de implementación el programa se cerró y no continuó siendo parte de la oferta pública, a pesar de los resultados reportados y de los aprendizajes acumulados que destacan como elementos central, las relaciones de colaboración mutua entre miembros de la comunidad y equipos profesionales (Olivares \& Reyes, 2016). Ello necesariamente invita a pensar en las continuidades y discontinuidades de las intervenciones propuestas, pues muchas veces no se logra avanzar desde el polo asistencial al transformativo, 
precisamente porque se interrumpen procesos y se detienen aprendizajes.

En definitiva, la PC sigue inmersa en un escenario complejo y tensionante que desata controversias entre quienes pensamos que el curso que ha tomado la disciplina requiere de reflexiones profundas (Wiesenfeld, 2014), que impliquen una re-lectura de los fundamentos que dieron origen a este modo de comprender y actuar con otros.

Si bien preocupan las limitaciones estructurales del contexto neoliberal, también se advierten las oportunidades presentes en los modos en que se implementan los programas, sobre todo cuando descomponemos procesos y distinguimos niveles. El ejercicio de realizar un zoom a programas en específico permite afinar la mirada y esbozar nuevas rutas para el quehacer comunitario, cargado de experiencias y aprendizajes que deben ser recuperados y socializados.

Ante este desafío, consideramos relevante tomar en cuenta las reflexiones del sociólogo Pierre Bourdieu cuando denuncia los objetivos del neoliberalismo, que se contraponen de manera radical a los objetivos y principios de la PC:

"En el nombre de este programa científico, convertido en un plan de acción política, un proyecto político inmenso está en marcha, aunque su condición de tal es negada porque parece ser puramente negativa. Este proyecto tiene como objetivo crear las condiciones en las que la "teoría" se puede realizar y puede funcionar: un programa de destrucción metódica de los colectivos" (Bourdieu, 1998). 


\section{Referencias}

Alfaro, J. (2007). Políticas sociales como condición de posibilidad para el desarrollo de prácticas en psicología comunitaria En J. Alfaro \& H. Berroeta (Eds.), Trayectoria de la psicología comunitaria en Chile: prácticas y conceptos (pp. 43-72). Valparaíso, Chile: Universidad de Valparaíso.

Alfaro, J. (2013). Psicología Comunitaria y Políticas Sociales: Institucionalidad y dinámicas de actores. Global Journal of Community Psychology Practice. Recuperado de: http://www.gjcpp.org/pdfs/alfaro-v4i2-20130613.pdf

Alfaro, J. y Zambrano, A. (2009). Psicología Comunitaria y políticas sociales en Chile. Revista Psicología \& Sociedade, 21(2), 275-282.

Arteaga, C. y Martuccelli, D. (2012). Neoliberalismo, corporativismo y experiencias posicionales. Los casos de Chile y Francia. Revista Mexicana de Sociología, 74(2), 275-302.

Bar-On, A. (1999). Social work and the missionary zeal to whip the heathen along the path of righteousness. British Journal of Social Work, 29(1), 5-26. Retrieved from http://bjsw.oxfordjournals.org.proxy.library.vcu.edu/content/29/1/5.short

Berroeta, H. (2011). Apuntes para una Intervención Psicosocial con Incidencia. Castalia, 13(19), $37-50$.

Berroeta, H. (2014). El quehacer de la Psicología Comunitaria: coordenadas para una cartografía. Psicoperspectivas, 14(2), 19-31.

Berroeta, H., Hatibovic, F. \& Asún, D. (2012). Psicología Comunitaria: prácticas en Valparaíso y visión disciplinar de los académicos nacionales. Polis, Revista Latinoamericana, 11(31), 335-354.

Bourdieu, P. (diciembre de 1998). The essence of neoliberalism. Le Monde Diplomatique. Recuperado de https://mondediplo.com/1998/12/08bourdieu?fbclid=IwAR152kFUKMiZuuH2Ve6A_0Q31 JgbPRxNWSjac5x-WwoobLFtpBweBSTknCs.

Brady, S. R. (2012). Discovering how community organizing leads to social change: Developing formal practice theory for social workers engaged in empowering community organizing (Doctoral dissertation, Virginia Commonwealth University). Disponible en ProQuest Dissertations and Thesis database. (UMI No. 3546921).

Brady, S., Schoeneman A. \& Sawyer, J. (2014). Critiquing and Analyzing the Effects of Neoliberalism on Community Organizing: Implications and Recommendations for Practitioners and Educators. Journal for Social Action in Counseling and Psychology, 6(1), $36-60$.

Burton, M. \& Kagan, C. (2003), Public policy initiatives and their impact on communities: Challenges for community psychology. Consultado en: $<$ www.compsy.org.uk/pub\%20pol\%20paper.pdf>.

Choudry, A., \& Shragge, R. (2011). Disciplining dissent: NGOs and community organizations. Globalizations, 8(4), 503-517. doi:10.1080/14747731.2011.585855

De la Maza, G. (2004). Políticas públicas y sociedad civil en Chile: el caso de las políticas sociales (1990-2004). Política, 43, 105-148.

De la Maza, G. (2011). Espacio público y participación ciudadana en la gestión pública en Chile: límites y posibilidades. Polis, 10(30), 45-75.

Esping-Andersen, Katzman y Wormald (2002). Why we Need a New Welfare State. Oxford: Oxford University Press.

Francescato, D., Tomai, M. \& Mebane, M. (2006). Psicología comunitaria en la enseñanza y la orientación. Experiencias de formación: presencial y online. Madrid: Narcea

Fuenmayor, J. (2014). Política pública en América Latina en un contexto neoliberal: Una revisión crítica de sus enfoques, teorías y modelos. Cinta de Moebio, 50, 39-52. 
Gainza, A. (2006). La entrevista en profundidad individual. En Canales, M. (Ed). Metodologías de la investigación social. Santiago de Chile: LOM.

Garretón, M.A. (2007). Del postpinochetismo a la sociedad democrática. Globalización y política en el bicentenario. Santiago: Random House Mondadori.

Garretón, M.A. (2013). Neoliberalismo corregido y progresismo limitado. Los gobiernos de la Concertación en Chile, 1990-2010. Santiago: ARCIS/CLACSO.

Glaser, B. \& Strauss, A. (1967). Discovery of grounded theory. Chicago: Aldine.

Giorgi, V. (2012). Entre el control tutelar y la producción de ciudadanía: aportes de la Psicología Comunitaria a las políticas de infancia En Alfaro, J., Sánchez, A. y Zambrano, A. Psicología Comunitaria y Políticas Sociales. Reflexiones y experiencias (pp.201-226). Buenos Aires: Paidós

Harvey, D. (2005). A Brief History of Neoliberalism. Oxford: Oxford University Press.

Hasenfeld, Y. \& Garrow, E. (2012). Nonprofit Human-Service Organizations, Social Rights and Advocacy in Neoliberal Welfare State. Social Service Review, 86(2), 295-322.

Iñiguez, L. (1999). Investigación y evaluación cualitativa: bases teóricas y conceptuales. Revista de Atención Primaria, 23(8), 496- 502.

Instituto de Políticas Públicas Expansiva-udp (2009). El Chile que viene. De dónde venimos, dónde estamos y a dónde vamos. Santiago: Ediciones UDP.

Krause, M. \& Jaramillo, A. (1998). Intervenciones Psicológico-Comunitarias en Santiago de Chile. Santiago, Chile: Pontificia Universidad Católica de Chile.

Krause, M., Jaramillo, A., Monreal, V., Carvacho, H., Torres, A. (2011). Historia de la Psicología Comunitaria en Chile. En M. Montero, I. Serrano-García (Eds.), Historias de la Psicología Comunitaria en América Latina (pp. 115-138). Buenos Aires: Paidós.

Laval. C. \& Dardot, P. (2013). La nueva razón del mundo. Ensayo sobre la sociedad neoliberal. Barcelona: Gedisa.

Martínez, M., Jaramillo, L., Santelices, M. P. \& Krause, M. (2009). Psychologie communautaire en Amérique Latine: Trajectoire historique et enjeux actuels, Pratiques Psychologiques, 15, 29-38.

Molina, C. (2006). Universalismo básico. Una nueva política social para América Latina. Washington, D.C.: Inter-American Development Bank..

Montero, M. (2004). Introducción a la psicología comunitaria: desarrollo, conceptos y procesos. Buenos Aires: Paidós.

Montero, M. (2009). Community Action and Research as Citizenship Construction. American Journal of Community Psychology, 43, 149- 161.

Montero, M. (2010). Unión de actores sociales, participación comunitaria y ética, en la ejecución de políticas públicas. En Hincapié, A. Sujetos políticos y acción comunitaria. Claves para una praxis de la psicología social y de la clínica social-comunitaria en América Latina (pp. 4566). Medellín: Universidad Pontificia Bolivariana.

Mullaly, B. (2007). The social work vision: A progressive view. In B. Mullaly, The new structural social work (pp. 44-69). Canada: Oxford Press. Ochsenius, C. (2005). Estrategias focalizadas y participativas de superación de la pobreza y desarrollo de la concertación pública local: El caso de Cerro Navia, Chile. Recuperado de http://www.innovacionciudadana.cl/portal/imagen/File/ochsenius.pdf

Nelson, G. (2013). Community Psychology and Transformative Policy Change in the Neo-liberal Era. American Journal of Community Psychology, 52(3-4), 211-223

Nelson, G., \& Prilleltensky, I. (2010). Community psychology: In pursuit of liberation and wellbeing. New York: Palgrave. 
Ornelas, J., Vargas-Moniz, M., \& Duarte, T. (2010). Community Psychology and Social Change: A Story from field of Mental Health in Portugal. Global Journal of Community Psychology Practice, 1(1), 21-31.

Olivares, B. \& Reyes-Espejo, M. I. (2016). Evaluación de un programa social en infancia desde los principios de la Psicología Comunitaria. Revista Latinoamericana de Ciencias Sociales, Niñez y Juventud, 14 (1), pp. 431-444.Ortiz, M. (2014). El perfil del ciudadano neoliberal: la ciudadanía de la autogestión neoliberal. Sociológica, 29(83), 165-200.

Olivares Espinoza, B., Winkler Müller, M. I., Reyes Espejo, M. I., Berroeta Torres, H., \& Montero Rivas, M. (2018). ¿Y si pensamos la comunidad con derechos? Psicología Comunitaria, derechos y políticas públicas. Una relación compleja. Universitas Psychologica, 17(2), 113. https://doi.org/10.11144/Javeriana.upsy17-2.pcdp

Olivares, B, Winkler, M., Reyes, M., Berroeta, H. \& Montero, M. (2018). ¿Y si pensamos la comunidad con derechos?: Psicología Comunitaria, derechos y políticas públicas. Una relación compleja. Universitas Psicologica. Vol 17 (1).

Quintal de Freitas, F. (2000). Voices from the South: The Construction of Brazilian Community Social Psychology. Journal of Community \& Applied Social Psychology, 10, 315-326.

Prasad, M. (2006). The Politics of Free Markets: The Rise of Neoliberal Economic Policies in Britain, France, Germany, and the United States. Chicago: University of Chicago Press.

Prilleltensky, I. (2001). Value-based praxis in community psychology: Moving toward social justice and social action. American Journal of Community Psychology, 29(5), 747-778.

Prilleltensky, I. (2004). Validez psicopolítica: el próximo reto para la psicología comunitaria. En: M. Montero, Introducción a la psicología comunitaria: desarrollo, conceptos y procesos. Buenos Aires: Paidós.

Pyles, L. (2009). Progressive community organizing: A critical approach for a globalizing world. New York: Routledge.

Reyes, M. I. (2007). Orientaciones ético-valóricas de la psicología comunitaria en Chile: análisis descriptivo de los artículos publicados en revistas nacionales entre 1993 y 2003. En J. Alfaro \& H. Berroeta (Eds.), Trayectoria de la psicología comunitaria en Chile: Prácticas y conceptos (pp. 109-148). Valparaíso, Chile: Editorial Universidad de Valparaíso.

Reyes, M.I., Olivares, B., Berroeta, H. y Winkler, M.I. (2016). Del discurso a las prácticas: Políticas sociales y psicología comunitaria en Chile. Polis, Revista Latinoamericana, 14(42), 387413. DOI: http://dx.doi.org/10.4067/S0718-65682015000300018.

Reyes, M.I., Jaramillo, K., Pizarro, P., Vergara, L., Navarrete, T. \& Yáñez, C. (en evaluación). La performatividad del Liderazgo Comunitario en las políticas públicas en el Chile: análisis de la oferta gubernamental y la percepción ciudadana.

Riesco, M. (2009). El modelo social chileno comienza a cambiar. Revista Internacional del Trabajo, 128(3), 311-330.

Rivera, G. (2017). Los procesos de influencia global/local en políticas públicas: una propuesta metodológica. Psicoperspectivas. Individuo y Sociedad, 16(3), 110-120.

Rodríguez, A. (2012). Aportes de la psicología comunitaria al campo de las políticas públicas sociales: el caso de Uruguay. En J. Alfaro, A. Sánchez \& A. Zambrano (Eds.), Psicología Comunitaria y Políticas Sociales. Reflexiones y experiencias (pp. 111-142), Buenos Aires: Paidos.

Rothman, J. (2007). Multi modes of intervention at the macro level. Journal of Community Practice, 15(4), 11-40. doi: 10.1300/J125v15n04

Rozas, G. (1995). Psicología Comunitaria en el desarrollo local y regional. Revista de Psicología Universidad de Chile, 5, 47-64.

Rozas, G. (2006). Lo social y la Identidad en las Políticas sociales. En G. Rozas \& J. Arredondo (Comps.). Identidad Comunidad y Desarrollo (pp. 159-178). Santiago: MIDEPLÁNFACSO. U. CHILE. Magíster de Psi Comunitaria. 
Shinn, M. (2007). "Waltzing with a Monster: Bringing Research to Bear on Public Policy". Journal of Social Issues, 63(1), 215-231.

Schram, S., Soss, J., Houser, L \& Fording, R. (2010). The Third Level of US Welfare Reform: Governm entality under Neoliberal Paternalism. Citizenship Studies, 14(6) 739-54. Recuperado de: http://www.bama.ua.edu/ rcfording/sshfcitizenshipstudies.pdf

Strauss, A. y Corbin, J. (2002). Bases de la investigación cualitativa. Técnicas y procedimientos para desarrollar la teoría fundamentada. Medellín: Universidad de Antioquia.

Wiesenfeld, E. (2014). La Psicología Social Comunitaria en América Latina: ¿Consolidación o crisis?. Psicoperspectivas, 13(2), 6-18

Received: $12 / 04 / 2018$

Accepted: 07/26/2019 